\title{
Mid-infrared transmission properties of amorphous germanium optical fibers
}

\author{
Priyanth Mehta ${ }^{1}$, Mahesh Krishnamurthi ${ }^{2}$, Noel Healy ${ }^{1}$, \\ Neil F. Baril ${ }^{2}$, Justin R. Sparks ${ }^{3}$, Pier J. A. Sazio ${ }^{1}$, \\ Venkatraman Gopalan ${ }^{2}$, John V. Badding ${ }^{3}$, \\ and Anna C. Peacock ${ }^{1}$
}

\footnotetext{
1 Optoelectronics Research Centre, University of Southampton, Southampton SO17 1BJ, UK 2 Department of Chemistry and Materials Research Institute, Pennsylvania State University, 16802 PA, USA

${ }^{3}$ Department of Chemistry and Materials Research Institute, Pennsylvania State University, 16802 PA, USA

acp@orc.soton.ac.uk
}

\begin{abstract}
Germanium optical fibers have been fabricated using a high pressure chemical deposition technique to deposit the semiconductor material inside a silica capillary. The amorphous germanium core material has a small percentage of hydrogen that saturates the dangling bonds to reduce absorption loss. Optical transmission measurements were performed to determine the linear losses over a broad mid-infrared wavelength range with the lowest loss recorded at $10.6 \mu \mathrm{m}$. The extended transmission range measured in the germanium fibers demonstrates their potential for use in mid-infrared applications.
\end{abstract}


Much of the recent progress in semiconductor photonics has leveraged off the highly developed silicon-on-insulator (SOI) based technologies. Following the development of the silicon Raman laser [1], a number of important optoelectronic devices have been demonstrated including switches [2], modulators [3] and an ultrafast optical oscilloscope [4]. More recently, lasing from the direct-gap transition in a germanium-on-silicon waveguide has been demonstrated [5]. Importantly, relative to silicon, germanium has an extended mid-infrared transmission window from $2-14 \mu \mathrm{m}$, a higher nonlinearity and superior electronic properties making it an advantageous material choice for a wide range of active waveguide devices. However, despite its desirable properties, few, if any, reports on the transmission losses of germanium waveguides can be found in the literature owing to the fabrication challenges facing these devices [6].

Semiconductor optical fibers are a class of waveguide that are currently generating much interest and germanium core fibers have been fabricated $[7,8]$. Fiberized devices offer a number of unique advantages in that, not only is the active semiconductor functionality now embedded seamlessly within the transmission medium, but they are robust, flexible and can be fabricated to have long electromagnetic interaction lengths. Unfortunately, owing to the high material loss in the early generations of these fibers, optical guiding in the germanium cores has yet to be demonstrated. In this paper we present investigations of the transmission properties of amorphous germanium core fibers fabricated using a high pressure microfluidic chemical deposition technique to completely fill the central pore of a silica capillary with the semiconductor material [7]. This simple and low cost procedure can be easily modified to fill a range of capillaries with differing core dimensions over several centimeters of length, and high production rates can be obtained by parallel fabrication of multiple fibers in a single deposition.

Optical loss measurements of the germanium fibers will be reported over a broad $2-11 \mu \mathrm{m}$ range of mid-infrared wavelengths for two different sized cores. Micro-Raman measurements have been used to establish the nature of the amorphous core which will be shown to have a small percentage of hydrogen. Significantly, the incorporation of hydrogen into amorphous materials is known to saturate the dangling bonds so that losses due to absorption are reduced [9]. The extended mid-infrared transmission range measured in the germanium waveguides demonstrates their potential to be used in areas ranging from broadband data transmission to biomedical applications.

To fabricate the germanium fibers, a mixture of germane and helium $\left(\mathrm{GeH}_{4} / \mathrm{He}\right)$ is forced to flow through the central hole of a fused silica capillary at high pressures $\sim 35 \mathrm{MPa}$. The deposition of semiconductor materials at such high pressures is permitted owing to the robust mechanical strength of 
fused silica capillaries [10]. For the fibers used in this paper the germanium is deposited at a relatively low temperature of $\sim 300^{\circ} \mathrm{C}$ so that the material grows in an amorphous state. This low temperature deposition is important as it allows for the interfacial layer of germanium to assume the pristine smoothness of the silica substrate $(0.1 \mathrm{~nm}$ root mean square surface roughness [11]) to minimize potential scattering losses at the core-cladding interface [12]. Furthermore, the low deposition temperature also suppresses the out diffusion of hydrogen, which occurs through the silica cladding, so that a percentage will remain in the semiconductor core material to passivate the dangling bonds. Similar hydrogenation of amorphous silicon core fibers has resulted in a large reduction of absorption losses [13], and thus we anticipate that this will provide an important route to improve the transmission of these fibers.

We have investigated two germanium fibers with core diameters of $5.6 \mu \mathrm{m}$ and $30 \mu \mathrm{m}$. A scanning electron microscope (SEM) micrograph of the $5.6 \mu \mathrm{m}$ core fiber provides confirmation of the complete filling of the capillary templates, as shown in Fig. 1(a), and in both cases the filled length was $\sim 3 \mathrm{~cm}$. To facilitate efficient free-space coupling of light into and out of the germa-
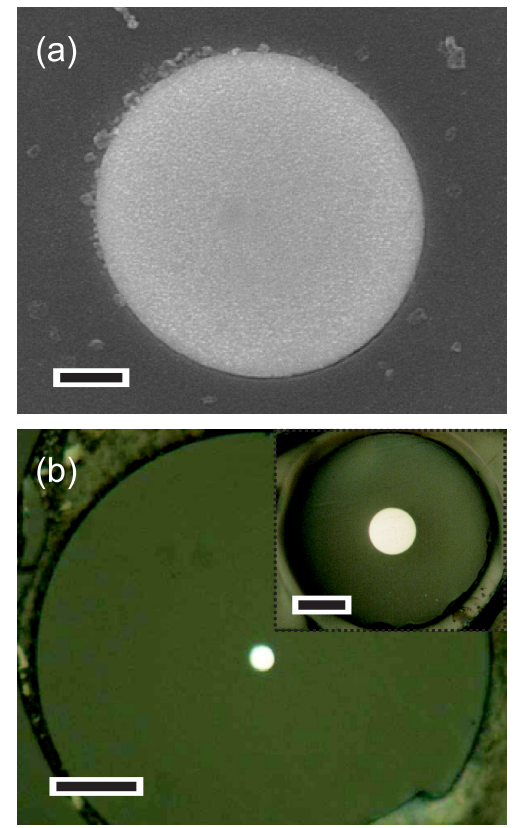

Figure 1: (a) SEM micrograph of the $5.6 \mu \mathrm{m}$ diameter core germanium fiber; scale bar $1 \mu \mathrm{m}$. (b) Optical image of the polished $5.6 \mu \mathrm{m}$ core fiber, mounted inside a thicker silica capillary; scale bar $20 \mu \mathrm{m}$. Inset shows the polished $30 \mu \mathrm{m}$ core fiber; scale bar $30 \mu \mathrm{m}$. 
nium fibers we mount them inside thicker silica capillary tubes and then use a standard polishing technique to finish the end faces. Fig. 1(b) shows an episcopic optical microscope image of the polished $5.6 \mu \mathrm{m}$ core fiber, with the $30 \mu \mathrm{m}$ fiber shown in the inset. The core materials were characterized using micro-Raman spectroscopy conducted on a Renishaw inVia system with a $633 \mathrm{~nm}$ HeNe laser source. A $100 \times$ objective was used to focus $250 \mu \mathrm{W}$ of power in a $0.4 \mu \mathrm{m}$ spot directly onto the polished germanium cores and a typical spectrum of the backscattered radiation is shown in Fig. 2. The Raman spectrum shows a broad peak, indicative of an amorphous material, around $280 \mathrm{~cm}^{-1}$, which is slightly shifted from the transverse optical Raman resonance of $278 \mathrm{~cm}^{-1}$ for amorphous germanium [9]. This observed shift is not unexpected as silica exhibits a different thermal expansion to germanium so that cooling from the elevated deposition temperatures will induce thermal mismatch stresses. Evidence of hydrogenation is provided by the peak at $\sim 1900 \mathrm{~cm}^{-1}$, as shown in the inset of Fig. 2, which is associated with the $\mathrm{Ge}-\mathrm{H}$ stretching mode. Although this stretching mode is typically split with a second vibration appearing at $\sim 2000 \mathrm{~cm}^{-1}$, our observation of only a single peak suggests that the hydrogen content is low [9]. Investigations are currently underway to increase the hydrogen content in these fibers, however, as the $\mathrm{Ge}-\mathrm{H}$ bond is much weaker than its $\mathrm{Si}-\mathrm{H}$ counterpart, even lower deposition temperatures are required to retain hydrogen in the material [13] and this in turn reduces the rate of decomposition of the precursor.

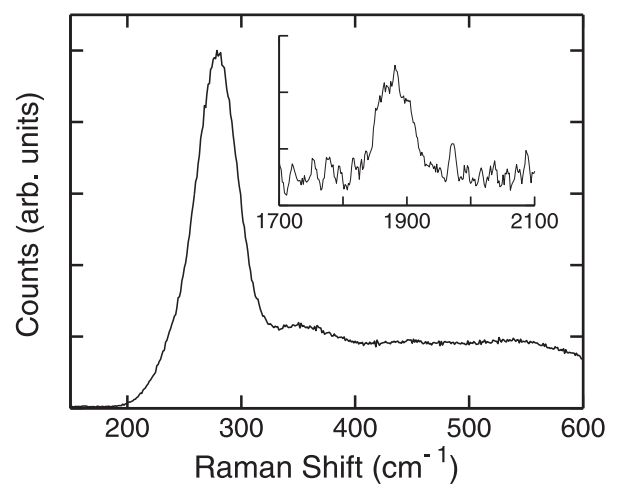

Figure 2: Raman spectrum of an amorphous germanium fiber. Inset indicates the presence of hydrogen.

The optical transmission properties of the germanium fibers were measured using various laser sources over the wavelength range $2-11 \mu \mathrm{m}$. In all cases the single pass measurement technique was used on fibers of length $\sim 5 \mathrm{~mm}$ to determine the average loss value at a given wavelength. This technique was deemed to be the most appropriate owing to there being a 
variation in the hydrogen content over the fiber lengths which led to significant deviations in the losses calculated via the cutback method. The varying hydrogen content is due to there being a temperature gradient across the furnace during the deposition and methods to generate a more uniform profile are being developed. The losses of the $5.6 \mu \mathrm{m}$ core fiber were measured over the wavelength range $2-2.4 \mu \mathrm{m}$ using a Radiantis Opium optical parametric oscillator (OPO) pumped via a Spectra-Physics Mai Tai laser operating at $80 \mathrm{MHz}$. Wavelengths below $2 \mu \mathrm{m}$ were not considered as the band edge absorption was too large and the OPO power above $2.4 \mu \mathrm{m}$ was too low for the transmission to be recorded accurately. Owing to the short, $250 \mathrm{fs}$, pulses delivered by this OPO system, low average input powers of $\sim 100 \mu \mathrm{W}$ were maintained to avoid nonlinear absorption associated with two-photon effects. The light was then launched into the core of the fiber via free-space coupling using a $40 \times$ microscope objective lens and collected via a second $40 \times$ objective lens. Piezo controlled nano-positioning stages were used to ensure precision coupling into and out of the fiber. The inset in Fig. 3(a) shows an example of the output beam profile imaged at $2 \mu \mathrm{m}$ on a Spricon Pyrocam III Series camera from which it is clear that the guided light is well confined to the high refractive index $(n \sim 4.1)$ core [14]. The output powers were measured using a PbSe preamplified detector and, after coupling and reflection losses are accounted for, the final transmission losses are presented in Fig. 3(a) showing a decrease in loss for increasing wavelength. This trend is consistent with our previous measurements for the losses in hydrogenated amorphous silicon fibers, in which scattering effects were deemed to be the major contributor [13]. Comparing these results with the $\lambda^{-4}$ dependence associated with Rayleigh scattering, shown by the fitted curve, the agreement is reasonable considering the band edge proximity and the difficulty in measuring the high losses in this particular wavelength regime. Nevertheless, these loss values are comparable to those of the early amorphous silicon optical fibers measured at $\sim 1.55 \mu \mathrm{m}$, which, as previously mentioned, have since been reduced by increasing the amount of hydrogen incorporation [13].

Loss measurements at the longer wavelengths of $3.39 \mu \mathrm{m}$ and $10.6 \mu \mathrm{m}$ were conducted on the $30 \mu \mathrm{m}$ diameter core fiber owing to the difficulty in coupling into the smaller core structure. The laser sources used at these wavelengths were both continuous wave $(\mathrm{CW})$, where at $3.39 \mu \mathrm{m}$ a HeNe laser was used with an input power of $\sim 12 \mathrm{~mW}$ and at $10.6 \mu \mathrm{m}$ a $\mathrm{CO}_{2}$ laser was used with an input of $\sim 30 \mathrm{~mW}$. In both cases the light was launched into the germanium core using a $25.4 \mathrm{~mm}$ focal length ZnSe lens and the output focussed onto a Laser Probe Inc. RK-5720 thermal power meter, with a RKP-575 detector head, using a $52 \times$ Ealing gold coated reflecting objective. Coupling into the core was facilitated by using a pyroelectric Electrophysics PV 320 camera to 

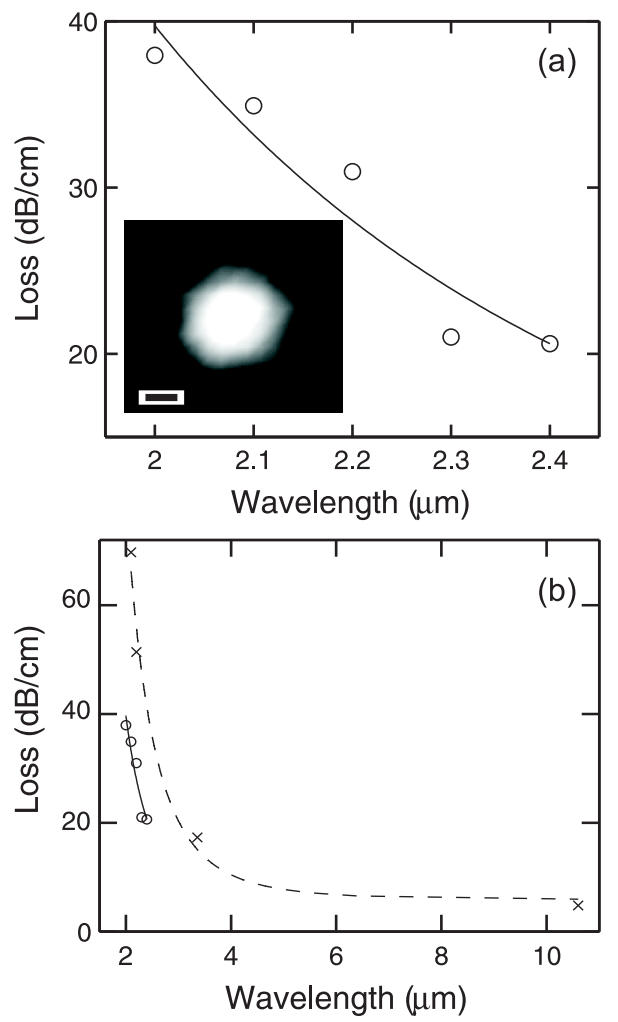

Figure 3: (a) Single pass transmission losses for the $5.6 \mu \mathrm{m}$ core germanium fiber together with an inset showing the guided output recorded at $2 \mu \mathrm{m}$; scale bar $4 \mu \mathrm{m}$. (b) Loss measurements for the $30 \mu \mathrm{m}$ core fiber (crosses) compared to those of the $5.6 \mu \mathrm{m}$ core fiber (circles).

image the guided output. The losses measured at these two wavelengths were $17.3 \mathrm{~dB} / \mathrm{cm}$ at $3.39 \mu \mathrm{m}$ and a much reduced value of $4.8 \mathrm{~dB} / \mathrm{cm}$ at $10.6 \mu \mathrm{m}$, which is significantly lower than previously reported losses for mid-infrared transmission in germanium $[15,16]$. In order to relate the loss values in this large core fiber with those of the smaller core fiber, measurements were also conducted around $2 \mu \mathrm{m}$ using the OPO and the results obtained over the entire wavelength range are plotted in Fig. 3(b) (crosses) together with the results of Fig. 3(a) (circles). The good agreement between the results for the $30 \mu \mathrm{m}$ core fiber and the $\lambda^{-4}$ fit (dashed line) provides further evidence that the losses in these fibers are dominated by scattering mechanisms. By comparing the results for the two fibers it is clear that, in this case, the smaller core fiber exhibits the lower material loss and this is likely to be due to either an increased hydrogen content or a more uniform distribution of hydrogen throughout the fiber length. Importantly, these results suggests 
that the low loss measured in the $30 \mu \mathrm{m}$ core germanium fiber at $10.6 \mu \mathrm{m}$ would be even lower in a fiber with a similar material quality to that of the $5.6 \mu \mathrm{m}$ core fiber. We anticipate that with such improvement in the material quality these germanium core fibers could lead to nonlinear devices in the $8-11 \mu \mathrm{m}$ regime, analogous to the current generation of silicon photonic devices that are restricted to operate at wavelengths below $\sim 6.5 \mu \mathrm{m}$.

We have demonstrated optical guiding in germanium core fibers over the broad mid-infrared wavelength region spanning $2-11 \mu \mathrm{m}$. Importantly, these results represent what we believe to be the most comprehensive range of loss measurements conducted in a germanium waveguide to date. By optimizing the deposition procedure to improve the germanium material quality and better understand the process of hydrogen incorporation, we anticipate that the losses in these fibers could be reduced over this entire wavelength range, as has similarly been achieved within silicon core fibers. The low loss measured at $10.6 \mu \mathrm{m}$ indicates that efficient device performance should be possible in this long wavelength regime, which is beyond the transmission window of silicon, opening up the potential for germanium fibers to find use in important applications in medicine and spectroscopy.

The authors acknowledge EPSRC (EP/G051755/1 and EP/G028273/1), NSF (DMR-0806860) and the Penn State Materials Research Science and Engineering Center (NSF DMR-0820404) for financial support. ACP holds a Royal Academy of Engineering fellowship. 


\section{References}

[1] O. Boyraz and B. Jalali, Opt. Express 12, 5269 (2003).

[2] V. R. Almeida, C. A. Barrios, R. R. Panepucci, M. Lipson, M. A. Foster, D. G. Ouzounov, and A. L. Gaeta, Opt. Lett. 29, 2867 (2004).

[3] A. Liu, L. Liao, D. Rubin, H. Nguyen, B. Ciftcioglu, Y. Chetrit, N. Izhaky, and M. Paniccia, Opt. Express 15, 660 (2007).

[4] M. A. Foster, R. Salem, D. F. Geraghty, A. C. Turner-Foster, M. Lipson, and A. L. Gaeta, Nature 456, 81 (2008).

[5] J. Liu, X. Sun, R. Camacho-Aguilera, L. C. Kimerling, and J. Michel, Opt. Lett. 35, 679 (2010).

[6] R. A. Soref, S. J. Emelett, and W. R. Buchwald, J. Opt. A: Pure Appl. Opt. 8, 840 (2006).

[7] P. J. A. Sazio, A. Amezcua-Correa, C. E. Finlayson, J. R. Hayes, T. J. Scheidemantel, N. F. Baril, B. R. Jackson, D.-J. Won, F. Zhang, E. R. Margine, V. Gopalan, V. H. Crespi, and J. V. Badding, Science 311, 1583 (2006).

[8] J. Ballato, T. Hawkins, P. Foy, B. Yazgan-Kokuoz, R. Stolen, C. McMillen, N. K. Hon, B. Jalali, and R. Rice, Opt. Express 17, 8029 (2009).

[9] D. Bermejo and M. Cardona, J. Non-Cryst. Solids 32, 405 (1979).

[10] C. R. Kurkjian, J. T. Krause, and M. J. Matthewson, J. Lightwave Technol. 7, 1360 (1989).

[11] P. J. Roberts, F. Couny, H. Sabert, B. J. Mangan, D. P. Williams, L. Farr, M. W. Mason, A. Tomlinson, T. A. Birks, J. C. Knight, and P. St. J. Russell, Opt. Express 13, 236 (2005).

[12] N. Healy, J. R. Sparks, P. J. A. Sazio, J. V. Badding, and A. C. Peacock, Opt. Express 18, 7596 (2010).

[13] L. Lagonigro, N. V. Healy, J. R. Sparks, N. F. Baril, P. J. A. Sazio, J. V. Badding, and A. C. Peacock, Appl. Phys. Lett. 96, 041105 (2010).

[14] D. H. Rank, H. E. Bennett, and D. C. Cronemeyer, J. Opt. Soc. Am. 44, 13 (1954). 
[15] W. S. C. Chang and K. W. Loh, Appl. Opt. 10, 2361 (1971).

[16] S. E. Plunkett, S. Propst, and M. S. Braiman, Appl. Opt. 36, 4055 (1997). 